\title{
Corporate Social Responsibility in der Marktwirtschaft - ein erfahrungswissenschaftlich begründetes Konzept ${ }^{*}$
}

\author{
HEIKO HOßFELD UND UTE SCHMIEL ${ }^{* *}$
}

Der Beitrag skizziert ein erfahrungswissenschaftlich fundiertes Konzept der CSR. Dabei fragen wir zunächst danach, wie Unternehmen handeln sollen und kommen zu dem Ergebnis, dass sie unter den Bedingungen einer Marktwirtschaft die Handlungsfreiheit anderer Wirtschaftssubjekte wahren sollen. In einem nächsten Schritt thematisieren wir, $\mathrm{ob}$ - und wie - ein solches Verständnis von CSR unter den Prämissen der ResourceDependence-Theorie realisierbar ist.

Schlagwörter: Verantwortung, CSR, Freiheit, genuine Unsicherheit, Macht, ResourceDependence-Theorie

\section{Corporate Social Responsibility in the Market Economy - a Concept Based on Empirical Science}

The article outlines a concept of CSR that is based on empirical science. In the context of a market economy, we define CSR as the preservation of freedom of choice. We also ask if - and how - such a concept can be realized under the assumptions of resource-dependence theory.

Keywords: CSR, Freedom, Uncertainty, Power, Resource-Dependence Theory

\section{Einleitung}

Unternehmen sollen verantwortlich handeln. So lautet Corporate Social Responsibility (CSR) als Handlungsregel formuliert. Eine solche Handlungsregel ist Bestandteil der Moral der deutschen Marktwirtschaftsordnung, die in gesetzlichen und außergesetzlichen Handlungsregeln konkretisiert ist. Green Washing und andere Formen unternehmerischen Handelns werfen jedoch die grundlegende Frage auf, ob CSR als Handlungsregel in der deutschen Marktwirtschaftsordnung adäquat konkretisiert ist. Diese Konkretisierung hat neben der ethischen eine erfahrungswissenschaftliche Dimension, wenn man wie in dem vorliegend vorgestellten Ansatz der kritisch-rationalen Methodologie folgt,

Beitrag eingereicht am 31.10.2014; nach doppelt verdecktem Gutachterverfahren überarbeitete Fassung angenommen am 01.10.2015.

Dr. Heiko Hoßfeld, Lehrstuhl für Betriebswirtschaftslehre, insbesondere Arbeit, Personal und Organisation, Fakultät Wirtschaftswissenschaften, Universität Duisburg-Essen, Universitätsstr. 12, D-45141 Essen, Tel. +49-(0)201-1833663, E-Mail: heiko.hossfeld@uni-due.de, Forschungsschwerpunkte: Legitimität managerialen Handelns aus theoretischer, empirischer und ethischer Perspektive, Manageriale Rhetorik, Employment Relations.

Prof. Dr. Ute Schmiel, Lehrstuhl für Unternehmensbesteuerung, Fakultät Wirtschaftswissenschaften, Universität Duisburg-Essen, Universitätsstr. 11, D-45141 Essen, Tel. +49-(0)2011832308, E-Mail: ute.schmiel@uni-due.de, Forschungsschwerpunkte: Ökonomische Steuerrechtsanalyse, ausgewählte methodologische und ethische Fragen der Betriebswirtschaftslehre. 
dass Sollen Können voraussetzt (vgl. Albert 1985: 98f.). Die Konkretisierung von CSR wird dann auch dadurch bestimmt, ob vor dem Hintergrund erfahrungswissenschaftlicher Markt- und Unternehmenstheorien eine solche Handlungsregel realisierbar ist.

Existierende Konkretisierungen von CSR berücksichtigen unserer Auffassung nach diese erfahrungswissenschaftliche Dimension nicht oder nach dem von uns eingenommenen kritisch-rationalen Wissenschaftsverständnis (vgl. Albert 2000: 7-16; Gadenne 2002) nicht adäquat: Diskursethisch begründeten Verantwortungskonzepten (vgl. Ulrich 2008: 427-499) fehlt ebenso wie anderen normativen Stakeholderansätzen (vgl. Freeman/Phillips 2002) weitgehend ein Bezug auf Markt- und Unternehmenstheorien. ${ }^{1}$ Der Business Case (vgl. Carroll/Shabana 2010) bzw. der instrumentelle Stakeholderansatz (vgl. Donaldson/Preston 1995) ignoriert ebenfalls in weiten Teilen die Bedingungen, unter denen Unternehmen agieren: Zwar erkennt er an, dass Stakeholder mit (legitimen) Ansprüchen gegenüber dem Unternehmen existieren, entproblematisiert diesen Umstand aber gleichzeitig, indem er unterstellt, dass eigennutzmaximierende Unternehmen diesen Ansprüchen ,automatisch" nachkommen würden, der Markt mithin unternehmerische Verantwortung adäquat reguliere. Dieser Ansatz dominiert heute den praktischen, wirtschaftlichen und politischen CSR-Diskurs (vgl. etwa Crane et al. 2008; Schreck 2012). Damit einher geht die im Diskurs dominierende Definition von CSR, die CSR als freiwilligen Beitrag der Unternehmen für soziale Belange und Umweltbelange charakterisiert (vgl. Europäische Kommission 2001; Loew et al. 2004; vgl. aber Europäische Kommission 2011). Die Kritik an einer ,automatischen“ Harmonie von Eigeninteresse und Gemeinwohl und die daraus resultierende Notwendigkeit eines normativen Regelwerks, welches die Eigennutzorientierung der Akteure so lenkt, dass zumindest Schäden des Gemeinwohls verhindert werden, verbindet unseren Ansatz mit der ökonomischen Ethik und Ordonomik. Anders als diese halten wir es jedoch nicht für ausreichend, Konflikte zwischen Eigeninteresse und Gemeinwohl ausschließlich auf (heuristisch eingesetzte) Dilemmastrukturen zurückzuführen und dabei den Homo oeconomicus als Zurechnungsschema zu verwenden (vgl. Homann/Blome-Drees 1992: 92-98; Homann/Suchanek 2005: 346-386, Suchanek 2007: 52-62, 177-188; LinHi 2009: 57-86; Homann 2014: 67-90, 101-105, 111-116; Pies/Hielscher 2014: 401403), um davon ausgehend diese Konflikte durch ein anreizkombatibel ausgestaltetes Regelwerk zu verringern.

Ziel unseres Beitrages ist es daher, ein Konzept der CSR zu entwickeln, das ethisch und erfahrungswissenschaftlich fundiert ist: Dies erfolgt vor der Grundprämisse des Systems einer Marktwirtschaft im Rahmen einer demokratischen Verfassung, also eines Systems, das grundsätzlich den Koordinationsmechanismus des Marktes - Akteure koordinieren sich über ihren Eigennutz weitgehend selbst - nutzt, anders als etwa ein planwirtschaftliches System. Der Ansatz soll die Verantwortung von Unternehmen vor dem Hintergrund übergreifender, ethisch legitimierter Marktziele konzipieren und dabei gleichermaßen die institutionellen Rahmenbedingungen, unter denen gewirtschaftet wird, in die Konzeption mit einbeziehen. Dabei bezieht sich unser Ansatz einerseits

So versteht Ulrich seinen Ansatz der integrativen Wirtschaftsethik als „Wirtschaftsethik obne Reflexionsstopp vor irgendwelchen empirischen und normativen Bedingungen der Marktwirtschaft" (Ulrich 2008: 133, Hervorhebung im Original). 
explizit auf das angeführte Verständnis einer Marktwirtschaftsordnung - und nimmt anders als etwa Ulrich keine inhaltliche Festlegung der Unternehmenstätigkeit vor (vgl. Ulrich 2008: 462-469) -, andererseits baut er auf realistischeren Annahmen über menschliches Handeln als Neoklassik, Business Case und ökonomischer Ethik bzw. Ordonomik auf und fragt in diesem Lichte explizit nach den Grenzen (und generellen Möglichkeiten) der Realisierung bzw. Durchsetzung von CSR.

Unser CSR-Konzept entwickeln wir im Folgenden vor der theoretischen Folie einer neoklassisch explizierten CSR, auf die (zumindest implizit) solche Ansätze rekurrieren, die von einer Harmonie von Eigen- und Gemeinwohl ausgehen (Kapitel 2). Im neoklassischen Paradigma ist mit der Gewinnmaximierungsnorm nicht nur eindeutig definiert, was Unternehmen tun sollen, um verantwortlich zu handeln, sondern darüber hinaus existieren hier keine Durchsetzungsprobleme, da Unternehmen in einem vollkommenen Konkurrenzgleichgewicht diese Norm verfolgen wollen und gleichzeitig keine strukturellen Bedingungen verhindern, dass sie dies auch können. Gegen diese Konzeption von CSR spricht jedoch, dass sie nicht realisierbar ist, weil Entscheidungssituationen durch genuine Unsicherheit (vgl. z.B. Witt 1987: 9-13; Buchanan/Vanberg 1991: 168f.; Ortmann 2009: 132) charakterisiert sind, die in der neoklassischen Theorie nicht abgebildet wird. Daher wird in Kapitel 3 nach den Inhalten der Unternehmensverantwortung in Märkten, die durch genuine Unsicherheit charakterisiert sind, gefragt. Dabei entwickeln wir aus den Zielen einer Marktwirtschaft die Wahrung der Handlungsfreiheit der Wirtschaftssubjekte als generelle Norm für verantwortliches Handeln, analysieren dabei aber gleichermaßen die Schwierigkeit, diese Norm unter Unsicherheit hinreichend zu konkretisieren. Weil genuine Unsicherheit jedoch nur einen Grund für Probleme bei der Durchsetzung von CSR-Normen darstellt, behandelt Kapitel 4 die Frage des Wollens und des Könnens. Zentral sind hier Aspekte der sozialen Einbettung von Unternehmen. Weil hierbei das Verhältnis von Freiheit und Verantwortung eine große Rolle spielt und Freiheit wiederum mit der Macht bzw. der Abhängigkeit der Wirtschaftssubjekte zusammenhängt, gründen wir hier unsere Überlegungen auf den Annahmen der ResourceDependence-Theorie. Der Aufsatz schließt mit einem Fazit (Kapitel 5).

\section{Neoklassisch explizierte CSR}

Die neoklassische Explikation von CSR beruht auf der Gleichsetzung von CSR mit der Gewinnmaximierung von Unternehmen, diese erfolgt vor dem Hintergrund des Marktziels „Pareto-Effizienz in einem allgemeinen Konkurrenzgleichgewicht“. Pareto-Effizienz wird in einem allgemeinen Konkurrenzgleichgewicht erreicht (vgl. Kleinewefers 2008: 42ff., 103f.; Varian 2011: 662-667), das Marktziel der Pareto-Effizienz wird also mit neoklassischen Handlungshypothesen und einer neoklassischen Markttheorie verbunden. Die Handlungshypothese der neoklassischen Theorie lautet, dass einzelne Wirtschaftssubjekte ihren Nutzen maximieren. Sie handeln objektiv rational, weil sie erstens konsistent handeln und zweitens perfektes, objektiv zutreffendes Wissen über die Welt haben (vgl. Vanberg 2005: 34 44). Dabei wird Nutzenmaximierung häufig auf die Maximierung des finanziellen Nutzens in Form von Konsum, Einkommen oder Vermögen beschränkt (vgl. Schmidt/Terberger 1997: 46-51). Generieren Wirtschaftssubjekte ihren finanziellen Nutzen als Unternehmenseigner, so besteht der Zweck von 
Unternehmen darin, durch Gewinnmaximierung - expliziert als Maximierung der positiven Ertragswertänderung - den (finanziellen) Nutzen der Anteilseigner zu maximieren (vgl. Schmidt/Terberger 1997: 46-51).

Nach neoklassischem Marktverständnis ist die Maximierung finanziellen Nutzens neben zahlreichen anderen Voraussetzungen eine Prämisse für das Vorliegen eines neoklassischen Konkurrenzgleichgewichts (vgl. Weise et al. 2005: 405f.; Vanberg 2003: 124f.; Varian 2011: 662-667). Versteht man Pareto-Effizienz als geeignetes Ziel der Marktwirtschaftsordnung, ist die Zulässigkeit der individuellen Nutzenmaximierung bzw. der Gewinnmaximierung von Unternehmen unter den Prämissen eines vollkommenen Konkurrenzgleichgewichts damit begründet, dass Unternehmen die Interessen anderer Wirtschaftssubjekte so berücksichtigen, dass gesamtwirtschaftliche Pareto-Effizienz realisiert wird. Grenze der Ertragswertmaximierung von Unternehmen ist der Nutzenverlust anderer Wirtschaftssubjekte, wobei im vollkommenen Markt keine externen Effekte existieren und Tauschbeziehungen nur eingegangen werden, wenn sie zum beiderseitigen Nutzen sind. Damit gilt für ein allgemeines Konkurrenzgleichgewicht:

- Unternehmen handeln verantwortlich, sofern sie Gewinne maximieren (expliziert als Maximierung der positiven Ertragswertänderung), denn dabei nehmen sie automatisch die Interessen anderer Wirtschaftssubjekte so in den Blick, dass gesamtwirtschaftliche Pareto-Effizienz erreicht wird. Darüber hinaus müssen sie nicht verantwortlich handeln.

- Zwischen eigen- und gesamtwirtschaftlichem Interesse besteht Harmonie: Unternehmen wollen in einem neoklassischen Paradigma verantwortlich in dem Sinne handeln, dass sie Gewinne maximieren.

- Unternehmen können unter neoklassischen Bedingungen nicht anders handeln, als Gewinne zu maximieren. Der Marktmechanismus zwingt sie zu dieser Form des Handelns: CSR-kompatible Maßnahmen wie die Auszahlung über dem Marktpreis liegender Löhne oder die umweltschonende Ressourcenverwendung erfordern (bei fortbestehender Gewinnmaximierungsprämisse) höhere Angebotspreise. Auf vollkommenen Märkten mit homogenen Gütern bedeutet dies für solche Unternehmen die Verdrängung aus dem Wettbewerb.

Neoklassisch explizierte CSR kann aus ethischer Sicht im Hinblick auf Pareto-Effizienz als Ziel der Marktwirtschaftsordnung kritisch hinterfragt werden (vgl. beispielsweise Ulrich 2008: 141-220). Wir beschränken uns jedoch hier auf erfahrungswissenschaftliche Kritik an der neoklassischen Handlungshypothese und Markttheorie (vgl. bereits Nutzinger 1992). Unserer Auffassung nach ist Pareto-Effizienz in einem vollkommenen Konkurrenzgleichgewicht bereits deshalb kein adäquates Marktziel, weil es dem kritisch-rationalen Adäquanzkriterium „Sollen impliziert Können“ (Albert 1985: 98f.) nicht genügt: Denn ein vollkommener Markt unter stochastischer Unsicherheit setzt voraus, dass der Entscheidende die Menge potentieller Zukunftslagen kennt. Stochastische Unsicherheit blendet damit die Probleme genuiner Unsicherheit aus (vgl. Schneider 1987: 1-6, 467-517; Witt 1987: 9-13; Buchanan/Vanberg 1991: 168f.). Nun ist jedes Modell aufgrund seiner immanenten Vereinfachung unrealistisch. Die besondere Problematik hinsichtlich eines vollkommenen Marktes unter Sicherheit bzw. stochastischer Unsicherheit liegt jedoch darin, dass CSR-relevante Probleme wie externe Effekte 
und Greenwashing erst in einer Welt mit genuiner Unsicherheit erklärt werden können. Solche Erfahrungstatbestände, die in vollkommenen Märkten unter Sicherheit oder stochastischer Unsicherheit nicht definiert sind, können nur widersprüchlich in Form sogenannter „Hybrid-Modell“ in vollkommene Märkte integriert werden. Diese setzen sich aus miteinander unvereinbaren Bestandteilen der realen Welt und der neoklassischen Welt zusammen (vgl. Richter/Furubotn 2010: 455f., 581-583), so dass hieraus jede beliebige Aussage gefolgert werden kann (vgl. Albert 1985: 16f., 56f.). Neoklassisch explizierte CSR ist unserer Auffassung nach bereits deshalb nicht begründet, weil neoklassisch fundierte Pareto-Effizienzin realen Märkten nicht erreicht werden kann.

\section{Inhalte der Unternehmensverantwortung unter genuiner Unsicherheit}

Weil unter genuiner Unsicherheit ein vollkommenes Konkurrenzgleichgewicht nicht erreicht werden kann, stellt sich die Frage nach den Inhalten der Unternehmensverantwortung jenseits vollkommener Märkte: Was sollen Unternehmen unter realen Marktbedingungen tun, um verantwortlich zu handeln? Hierzu gehört auch die Frage, ob sich jenseits eines allgemeinen Konkurrenzgleichgewichts gesamtwirtschaftliche Pareto-Effizienz auf eine Art erzielen lässt, die eine Gleichsetzung von CSR mit Gewinnmaximierung unter genuiner Unsicherheit legitimiert. Zur Beantwortung dieser Fragen bedarf es des Rückgriffs auf Theorien, für die genuine Unsicherheit konstitutiv sind.

In Abgrenzung zur neoklassisch fundierten Hypothese über das Handeln von Wirtschaftssubjekten werden hier unter Berücksichtigung genuiner Unsicherheit die folgenden Hypothesen über das Handeln von Wirtschaftssubjekten vertreten: Wirtschaftssubjekte verfügen nicht über vollkommenes, perfektes Wissen über die Welt, sondern nur über unvollständiges Wissen. Dies resultiert sowohl aus der Vielzahl möglicher Handlungsalternativen als auch daraus, dass zum Entscheidungszeitpunkt die Menge möglicher Handlungsalternativen nicht feststeht (vgl. Witt 1987: 9-30, 104-148). Letzteres ist auch eine Konsequenz daraus, dass Wirtschaftssubjekte Handlungsmöglichkeiten nicht nur erkennen, sondern durch kreatives Handeln auch schaffen (vgl. Witt 1987: 930, 104-149; Buchanan/Vanberg 1991: 168-171). Vor diesem Hintergrund ist objektiv rationales Handeln nicht möglich, Wirtschaftssubjekte können zwar konsistent handeln, aber lediglich gemäß ihrem subjektiven Wissen über die Welt (und damit subjektiv rational, vgl. Vanberg 2005: 34-44). Sie können allenfalls ihren subjektiv wahrscheinlichen Nutzen, ihre (finanziellen) Nutzenerwartungen, maximieren („Nutzenerwartungen“ wird in Anlehnung an Wegner 2012: 104-111 gewählt, der von Vorteilserwartung spricht). Ob diese Erwartungen erfüllt werden, ist jedoch ,genuin unsicher“. Mit Nutzenerwartungen ist also nicht der auf stochastischer Unsicherheit basierende Erwartungswert des Nutzens gemeint. Es ist vielmehr durchaus denkbar, dass Wirtschaftssubjekte ex post positiv oder negativ überrascht werden (vgl. Schneider 1987: 2). Selbst unter der (kontrafaktischen) Annahme, dass alle Wirtschaftssubjekte ihre finanziellen Nutzenerwartungen maximieren, existiert aufgrund genuiner Unsicherheit keine objektiv rationale Zielgröße, weil diese nicht in Zielgrößen erfasst werden kann. Daher können auch Unternehmen allenfalls Gewinnerwartungen maximieren. Hier zeigt sich ein elementarer Unterschied zur ökonomischen Ethik und Ordonomik, die als Analyseinstrument objektivrationales Handeln zugrunde legen (vgl. Homann/Blome-Drees 1992: 92-98; Homann/Suchanek 2005: 367-380; Homann 2014: 101-105; Homann/Lütge 2013: 
65-70; Pies et al. 2009: 378-388). Problematisch im Hinblick auf die fehlende empirische Bewährung ist dabei aus unserer Sicht weniger die angenommene Eigennutzorientierung als vielmehr die mit objektiver Rationalität verbundene Annahme über das perfekte Wissen der Wirtschaftssubjekte (vgl. hierzu Homann/Suchanek 2005: 380-383; Pies/Hielscher 2014). Unter genuiner Unsicherheit kann gesamtwirtschaftliche ParetoEffizienz damit allenfalls zufälliges Ergebnis eines Marktes sein. Es kann nicht verhindert werden, dass Wirtschaftssubjekte durch eigenes Handeln oder durch das Handeln anderer (finanzielle) Nutzenverluste erleiden (vgl. Wegner 2012: 136).

Es existiert also kein erfahrungswissenschaftlicher Zusammenhang zwischen der individuellen Gewinnmaximierung - allgemeiner: der individuellen Vorteilsverfolgung und dem Gemeinwohl im Sinne einer (finanziellen) Wohlstandsmaximierung: „The market economy, as an aggregation, neither maximizes nor minimizes anything" (Buchanan/Vanberg 1991: 181, Hervorhebung im Original). Dies ist vor allem auch deshalb der Fall, weil unter genuiner Unsicherheit Wirtschaftssubjekte keineswegs im Rahmen der Maximierung ihrer individuellen Nutzenerwartungen ,automatisch“ die Schädigung anderer Wirtschaftssubjekte vermeiden. Ein wichtiger Grund hierfür besteht in der Existenz negativer externer Effekte (z.B. Umweltschädigungen), deren Kosten für die Betroffenen nicht über den Marktmechanismus abgegolten werden. Ein zweiter Grund liegt in Informationsasymmetrien, wenn den Betroffenen nicht bekannt wird, dass sie geschädigt werden oder von wem sie geschädigt werden. Ein dritter Grund liegt in Machtasymmetrien, die in Kapitel 4 genauer behandelt werden.

In realen Märkten haben Unternehmen also durchaus auch jenseits der Gewinnmaximierung Verantwortung gegenüber den Anspruchsgruppen der Unternehmung, sofern Verantwortung so verstanden wird, dass Akteure für ihre Handlungen - und damit auch für die Konsequenzen ihres Tuns - Rechenschaft abzulegen haben (vgl. Heidbrink 2011; Nida-Rümelin 2011: 25). Über die konkreten Inhalte und die Reichweite der Verantwortung von Unternehmen existieren in der CSR-Forschung unterschiedliche Konzepte (für einen Überblick vgl. Carroll 1999; 2008). Dabei zeigt allerdings eine Studie von Dahlsrud (2008), die mittels einer Inhaltsanalyse 37 unterschiedliche Definitionen von CSR im internationalen Kontext identifiziert, dass viele Definitionen von CSR keine konkreten Inhalte von Unternehmensverantwortung benennen, sondern CSR eher als soziales Phänomen beschreiben, etwa, indem sie empirisch auftretende Gegenstandsbereiche der CSR - Umwelt, Soziales und/oder Wirtschaft - identifizieren und unterscheiden. Zu den wohl bekanntesten Modellen zur Strukturierung unternehmerischer Verantwortung zählt in diesem Kontext die Systematik von Carroll (1991, vgl. auch Aßländer 2010). In der sogenannten „Pyramid of Corporate Social Responsibility“ werden vier Arten von Verantwortlichkeiten unterschieden, wobei die ökonomische Verantwortung (,,be profitable“) an den Fuß der Pyramide gesetzt wird. Darauf aufbauend folgen die legale Verantwortung (,obey the law"), die ethische Verantwortung (,be ethical") und zuletzt, ganz oben, die philanthropische Verantwortung (,,be a good corporate citizen"), die sich in karitativen Tätigkeiten (etwa Spenden) ausdrückt (vgl. Carroll 1991: 42). Diesem Konzept mangelt es jedoch sowohl an einer ausreichenden ethischen Fundierung (vgl. hierzu etwa Aßländer/Löhr 2010: 17f.) als auch an der Verortung der Unternehmensverantwortung im Rahmen der Ziele einer Marktordnung. Ähnliches gilt für das im (vor allem angloamerikanischen) wissenschaftlichen Diskurs 
dominierende CSR-Konzept, den Stakeholderansatz (vgl. Freeman 1984, zur Bedeutung dieses Ansatzes z.B. Donaldson/Preston 1995; Dahlsrud 2008; Aguinis/Glavas 2012). Dieser hat sich im Laufe der Zeit in (mindestens) drei unterschiedliche Theoriestränge ausdifferenziert (vgl. Donaldson/Preston 1995). Vor allem im Praktikerdiskurs sind der deskriptive und instrumentelle Stakeholderansatz (bzw. der sogenannte Business Case, vgl. Carroll/Shabana 2010) von Bedeutung. Diese betonen, warum Unternehmen die Interessen ihrer Stakeholder aus pragmatischen Gründen berücksichtigen (sollten). Die Forschung konzentriert sich hier dementsprechend auf den Zusammenhang zwischen CSR und Unternehmenserfolg (für einen Überblick vgl. Aguinis/Glavas 2012), nicht jedoch auf die ethische Begründung von Verantwortung. Eine solche wurde erst später im sogenannten normativen Stakeholderansatz nachgeliefert. Hierbei werden etwa moralische Ansätze von Kant (vgl. Evan/Freeman 1988, Waldkirch 2008: 7) oder Aristoteles (vgl. Wijnberg 2000) zur Begründung der Berücksichtigung von Stakeholderinteressen herangezogen und damit der Konflikt zwischen Ökonomie und Moral betont (vgl. hierzu auch Wieland 2007: 88f.; aber auch Freeman/Phillips 2002).

Im Folgenden soll daher ein Konzept der CSR entwickelt werden, das die Frage nach den Inhalten der Unternehmensverantwortung auf Grundlage der Voraussetzungen und Ziele eines Systems der Marktwirtschaft begründet und konkretisiert. Dabei verstehen wir grundsätzlich den Markt als eine Form der Produktion und Verteilung von Gütern, die auf dem Eigeninteresse der beteiligten Individuen beruht. Grundidee und Grundmechanismus des Marktes ist es, dass Akteure nicht unmittelbar das Gemeinwohl steigern, sondern mittelbar, indem der Marktmechanismus Anreize setzt, Leistungen zu erstellen, die für andere Wirtschaftssubjekte von Nutzen sind. Dass dabei mittelbar auch die Interessen der anderen Wirtschaftssubjekte berücksichtigt werden, ist also nicht auf die „unsichtbare Hand des Marktes“ zurückzuführen, sondern darauf, dass Wirtschaftssubjekte die Interessen ihrer Tauschpartner berücksichtigen müssen, um selbst Vorteile zu erlangen (vgl. Buchanan/Vanberg 1991: 182). Ein praktikables Konzept der CSR sollte unserer Auffassung nach diesem Marktwirtschaftsverständnis Rechnung tragen. Deshalb ist es nicht sinnvoll (wie bei Ulrich 2008: 466, im Original teilweise hervorgehoben) an Unternehmen den Anspruch zu stellen, den Unternehmenszweck an einer „Vision der lebenspraktischen Werte, die geschaffen werden sollen, seien es (...) solche auf der Ebene der menschlichen Lebengrundlagen oder solche auf der Ebene der Erweiterung der menschlichen Lebensfülle" zu orientieren. Dies erforderte ein anderes Wirtschaftssystem. Die Diskussion eines solchen kann zwar fruchtbar sein, wir beschränken uns hier aber auf das System der Marktwirtschaft, da es einerseits das System ist, welches wir in westlichen Demokratien vorfinden. Andererseits gibt es gute Gründe, die für dieses System sprechen (vgl. etwa Boettcher 1974: 9; Schlecht 1990; Vanberg 2006). Dabei hat die auf dem Eigennutz der Akteure aufbauende freie Marktwirtschaft erstens einen systembedingten Vorteil: Eine Wirtschaftsordnung, die dafür sorgt, dass die Akteure freiwillig das tun, was im Sinne des Systems wünschenswert ist, spart sich (einige) Kosten der Kontrolle und der Fremdanreize. Zweitens ermöglicht eine Wirtschaftsordnung, die auf dem Eigennutz der Akteure aufbaut, grundsätzlich ein übergreifendes gesellschaftliches Ziel demokratischer Verfassungen: Die Handlungsfreiheit der Wirtschaftssubjekte (vgl. zur Freiheitssicherung durch die Marktwirtschaftsordnung 
bereits Homann/Blome-Drees 1992: 47-50; Suchanek 2007: 97-116; differenzierter: Homann/Lütge 2013: 46-50; Homann 2014: 97-100; Pies/Hielscher 2014: 407f.).

Weil aber die Wirtschaftssubjekte kein genuines Interesse an der Nutzensteigerung ihrer Tauschpartner haben, sondern lediglich ein instrumentelles, bedarf es eines normativen Regelwerks, welches die Eigennutzorientierung der Akteure auf eine Weise lenkt, in der das Gemeinwohl zumindest nicht zu Schaden kommt. Wie aber lässt sich dieses Gemeinwohl definieren, wenn eine (finanzielle) Gemeinwohlmaximierung gar nicht möglich ist? Hierzu greifen wir die Argumentation von Buchanan auf, der zum einen kein finanzielles Marktziel als ethische Leitlinie zugrunde legt, sondern die Freiheit der Akteure. Freiheit ist ebenso ein gesellschaftlicher Grundwert (vgl. etwa Streit 1988; Falk 2012) wie ein zentraler Beitrag einer Marktwirtschaftsordnung zum Gemeinwohl und gleichzeitig Voraussetzung für das Funktionieren von Märkten (vgl. Vanberg 1997). Zum anderen bezieht Buchanan Pareto-Effizienz nicht auf gesamtwirtschaftliche Zustände, sondern auf den jewveiligen Tausch: Ein realisierter Tausch reflektiert demzufolge ParetoEffizienz, sofern Wirtschaftssubjekte Handlungsfreiheit haben (vgl. Brennan/Buchanan 1985: 23ff., auch Nutzinger 1992: 61). Denn wenn Wirtschaftssubjekte Handlungsfreiheit haben, wird es nur dann zu einem Tausch kommen, wenn jeder Tauschpartner gemäß seiner subjektiven Einschätzung durch den Tausch besser gestellt wird, als er ohne Tausch stünde. Wenn Wirtschaftssubjekte an einem Tausch interessiert sind, müssen sie die Interessen des Tauschpartners insoweit berücksichtigen, wie jener seine Zustimmung zum Tausch an das Erreichen seiner Interessen bindet. Weil der Tauschpartner aufgrund seiner Handlungsfreiheit seine Interessen selbst vertreten wird, ist gewährleistet, dass diese mittelbar Berücksichtigung finden. Unmittelbar müssen Wirtschaftssubjekte die Interessen anderer Wirtschaftssubjekte nicht verfolgen (ähnlich Vanberg 2007: 204, 215; 2010: 107-111). Die mögliche Verweigerung der Zustimmung ist demzufolge das Instrument, mit dem Wirtschaftssubjekte die Berücksichtigung ihrer Interessen erreichen können.

Allerdings haben Wirtschaftssubjekte in realen Märkten nicht immer die Möglichkeit, ihre Zustimmung zu verweigern. Es ist nämlich keineswegs so, dass der Markt automatisch Wahl- bzw. Handlungsfreiheit produziert (vgl. hierzu das folgende Kapitel). Dies wäre nur dann der Fall, wenn Freiheit mit Einwilligung gleichgesetzt würde, also aus dem Umstand, dass in einer Marktwirtschaft Verträge grundsätzlich freiwillig abgeschlossen werden, abgeleitet würde, dass dieser Vorgang ein Ausdruck der Handlungsfreiheit individueller und korporativer Akteure ist (vgl. Peter 2004: 164). Der realisierte Tausch reflektiert jedoch unserer Auffassung nach Pareto-Effizienz nicht bereits bei einer Abwesenheit von Zwang (negativer Freiheitsaspekt), sondern erst dann, wenn Wirtschaftssubjekte die Freiheit haben, zu handeln (positiver Freiheitsaspekt). Diese Freiheit ist in realen Märkten jedoch an einige Voraussetzungen geknüpft: Zum einen müssen Wirtschaftssubjekte aufgrund der Abwesenheit von Zwang die Unterlassungsalternative auch tatsächlich wählen können. Dies setzt zumindest eine existenzsichernde Ressourcenausstattung natürlicher Personen voraus (vgl. zu dieser Deutung positiver Freiheit als Möglichkeitskonzeption und zu weiteren Deutungen von negativer bzw. positiver Freiheit Sen 1988; Ladewig 2011: 84ff.; van Aaken 2012: 86-90). Zum anderen müssen Wirtschaftssubjekte zumindest grundlegend informiert sein; ihre Fähigkeit zu 
handeln darf nicht durch (aufgrund von Informationsasymmetrien mögliches) opportunistisches Handeln beeinträchtigt werden. Außerdem reflektiert ein realisierter Tausch bezogen auf die von dem Tausch betroffenen Personen Pareto-Effizienz nur dann, wenn von diesem keine negativen externen Effekte ausgehen bzw. wenn adäquate Regeln zum Umgang mit negativen externen Effekten berücksichtigt wurden. Auf den jeweiligen Tausch bezogene Pareto-Effizienz und die damit verknüpfte Zulässigkeit, eigennutzorientiert zu handeln, setzt damit bereits adäquate Tauschregeln über die $\mathrm{Zu}$ lässigkeit eigennutzorientierten Handelns und dessen Grenzen sowie Regeln zur Einhaltung dieser Tauschregeln voraus (vgl. bereits Brennan/Buchanan 1985: 24). Elementar im Rahmen dieser Tauschordnung sind Regeln zur Wabrung der negativen und positiven Handlungsfreibeit.

Unter genuiner Unsicherheit ist es nun allerdings nicht möglich, more geometrico aus dem Prinzip der Handlungsfreiheit eine adäquate Tauschordnung zu deduzieren, zumal ein dem Pareto-Kriterium vergleichbares Beurteilungskriterium für die Herleitung einer adäquaten Tauschordnung fehlt: Denn wenn die auf den individuellen Tausch bezogene Pareto-Effizienz, mit der die Zulässigkeit eigennutzorientierten Handelns begründet wird, bereits adäquate Tauschregeln voraussetzt, können diese nicht mit der auf den Tausch bezogenen Pareto-Effizienz begründet werden (vgl. Brennan/Buchanan 1985: 25-31; Vanberg 1997: 712-715). Unserer Auffassung nach besteht nur die Möglichkeit, Vorschläge für Tauschregeln zu erarbeiten, mit realisierbaren Alternativen zu vergleichen und so kritisch zu überprüfen (vgl. Albert 1985: 218-229; für das Beispiel der Steuervermeidung: Schmiel/Hoßfeld 2014). Nach hier vertretener Auffassung ist dabei ausgehend von den zu regelnden Sachverhalten auf ethische Konzeptionen zur Handlungsfreiheit und weitere relevante Prinzipien zu rekurrieren und auf die zu untersuchenden Probleme zu applizieren. Weil hier in Anlehnung an Albert die Auffassung vertreten wird, dass „Sollen Können impliziert““ (Albert 1985: 98f.), ist dabei auch auf erfahrungswissenschaftliche Hypothesen zu rekurrieren und zu prüfen, ob eine Handlungsregel realisierbar ist. Mit Blick auf (gerade unter genuiner Unsicherheit zu erwartende) neue Sachverhalte und die Vorläufigkeit ethischen und erfahrungswissenschaftlichen Wissens wäre es unserer Auffassung nach eine Überschätzung des Wissens, würde man davon ausgehen, eine vollständige Tauschordnung aus dem Ziel der Handlungsfreiheit deduzieren zu können (vgl. Vanberg 2003: 129-133). Wenn aber nicht davon auszugehen ist, dass eine vollständige Tauschordnung deduziert werden kann, so ist offensichtlich, dass auch keine vollständige gesetzlich kodifizierte Tauschordnung möglich ist. Letzteres gilt zusätzlich besonders vor dem Hintergrund, dass dem nationalen Gesetzgeber die Kompetenz fehlt, grenzüberschreitendes unternehmerisches Handeln umfassend zu regeln (vgl. auch Burkatzki/Löhr 2008: 13-16). Weil eigennutzorientiertes Handeln die Einhaltung von Tauschregeln voraussetzt und von der Unvollständigkeit gesetzlicher Regeln auszugehen ist, reicht es unserer Auffassung nach nicht aus, Unternehmen nur für die Einhaltung gesetzlicher Regeln verantwortlich zu machen und die Ausnutzung gesetzlicher Lücken uneingeschränkt zuzulassen (vgl. auch Vanberg 2007: 215). Vielmehr sollen Unternehmen die positive und negative Handlungsfreiheit ihrer Tauschpartner und der von externen Effekten Betroffenen sowie andere elementare Prinzipien der Tauschordnung wahren (vgl. zur Verknüpfung von Freiheit und normativem Stakeholderansatz Freeman/Phillips 2002). Dies gilt auch dann, wenn eine 
gesetzliche Regelung fehlt bzw. wenn dem nationalen Gesetzgeber die Regelungskompetenz entzogen ist (vgl. z.B. van Aaken 2012: 88-90). Denn nur soweit Handlungsfreiheit des Tauschpartners vorliegt, kann dieser seine Zustimmung zum Tausch an die Realisierung der eigenen Interessen binden. Sofern die Handlungsfreiheit des Tauschpartners nicht ausreicht, sind dessen Interessen hingegen unmittelbar in den Blick zu nehmen (vgl. ebd.: 90). Vor diesem Hintergrund ist aus unserer Sicht der systematische Ort der Moral nicht allein die Rahmenordnung (vgl. Homann/Blome-Drees 1992: 20-53, relativierend: 118-149, 159-167; Homann 2002: 27-35, relativierend: 35-42), sondern auch die Handlungsebene der Akteure.

Wirtschaftssubjekte, die ihrer Verantwortung in dieser Weise nachkommen, verhalten sich damit nicht nur ethisch verantwortlich, sondern auch ökonomisch verantwortlich: Sie handeln im Sinne einer Marktordnung, denn die individuelle Handlungsfreiheit der Wirtschaftssubjekte stellt sowohl Ziel als auch Funktionsbedingung für ein System der Marktwirtschaft dar. Daher ist es nicht sinnvoll, ökonomische Verantwortung auf die Gewinnerzielung zu reduzieren und von der ethischen abzugrenzen (vgl. aber Carroll 1991). Ein weiterer Unterschied zu Carrolls CSR-Pyramide: Vor dem von uns verwendeten Verantwortungsbegriff können Unternehmen gegenüber Dritten nur in ökonomischer, rechtlicher und ethischer Hinsicht rechenschaftspflichtig sein; darüber hinausgehendes Engagement im Sinne einer philanthropischen Verantwortung kann somit nur frei sein und nicht eingefordert werden (vgl. Kleinfeld/Henze 2010: 69). Damit erfasst unser Ansatz ganz bewusst einen, vor allem im angloamerikanischen Raum sehr populären Zweig der CSR nicht. Die sogenannte Spendenethik (vgl. Aßländer 2010, bereits ablehnend: Friedman 1970, ebenfalls beispielsweise Suchanek 2007: 124ff.; Lin-Hi 2009: 23-29). Denn der hier vorgestellte Verantwortungsbegriff erstreckt sich primär auf den Tausch mit den Stakeholdern der Unternehmung.

Auch die Unterscheidung zwischen ethischer und rechtlicher Verantwortung erscheint wenig trennscharf, da die Rechtsordnung einer Marktordnung ebenfalls ethisch legitimiert bzw. begründet ist. Vor dem Hintergrund genuiner Unsicherheit macht jedoch zumindest die analytische Trennung der beiden Sinn, da sie verdeutlicht, dass es aufgrund unvollständiger Regelwerke gar nicht möglich ist, Verantwortung auf legale Aspekte zu reduzieren. Dieser Umstand ist auch im praktischen und wissenschaftlichen CSR-Diskurs lange bekannt; tatsächlich konzentriert sich insbesondere die Praxis auf die Entwicklung solcher ,außergesetzlicher" Regelungen in Form von Standards und Kodizes (z.B. ISO 26000, Global Compact, OECD-Leitsätze für multinationale Unternehmen, vgl. Aßländer 2011: 115). Dabei gilt spätestens, seit die Europäische Kommission (2001: 7) in ihrem Grünbuch CSR als ein Konzept definierte, das ,auf freiwilliger Basis soziale Belange und Umweltbelange" berücksichtigt, diese Freiwilligkeit auch im europäischen CSR-Diskurs als wesentliches Merkmal von CSR. ${ }^{2}$ Konzeptionelle bzw. theoretische Unterstützung erhält diese Konkretisierung von CSR durch den Business Case bzw. instrumentellen Stakeholderansatz, der auf den ökonomischen Nutzen von

2 Aßländer/Löhr (2010: 15) begründen diese Unverbindlichkeit damit, dass das Grünbuch die Unternehmen nicht abschrecken sollte. In der neuen Definition der Europäischen Kommission (2011: 9) ist hingegen nicht mehr vom freiwilligen Engagement die Rede, und die Kommission signalisiert, dass sie nun auf die „Kombination aus freiwilligen Maßnahmen und nötigenfalls ergänzenden Vorschriften“ setzt. 
CSR fokussiert und dabei unterstellt, dass Unternehmen bereits aus eigenem Interesse heraus einen Anreiz haben, verantwortungsvoll zu handeln (für einen Überblick vgl. Kurucz et al. 2008; Devinney 2009). Dies erweckt den Eindruck, als wäre ein (externer) Zwang zu verantwortungsvollem Handeln nicht nötig.

Einen ähnlichen Fokus auf freiwillige CSR-Lösungen hat die Ordonomik (und in Teilen auch die ökonomische Ethik). Ihre Vertreter unterstellen zwar keine grundsätzliche Übereinstimmung von Eigen- und Gemeinwohlinteresse und verpflichten Wirtschaftssubjekte sogar dazu, in die gesellschaftliche Zusammenarbeit zum gegenseitigen Vorteil zu investieren, dies wird für Unternehmen allerdings mit einer moralischen Pflicht zur Gewinnmaximierung gleichgesetzt (Homann/Blome-Drees 1992: 38; Suchanek 2007: 134-152, 79-87, Lin-Hi 2009: 93, 126). Ferner begrenzen sie die Übernahme von Verantwortung durch Unternehmen: Weil Unternehmen zur gesellschaftlichen Freiheit beitragen, muss die Übernahme von Verantwortung grundsätzlich anreizkompatibel sein (Lin-Hi 2009: 87-137). Insbesondere die Ordonomik entproblematisiert diesen Umstand aber, indem sie gesellschaftliche Missstände als soziale Dilemmata rekonstruiert, die aufgrund eines gemeinsamen Regelinteresses aufgelöst werden können (vgl. Pies/Hielscher 2008: 342). Dies geschieht unter Rückgriff auf das spieltheoretische Gefangenendilemma. Dabei werden soziale Dilemmata definiert als Situationen der kollektiven Selbstschädigung (vgl. Will/Hielscher 2014: 226): Eine für alle Akteure vorteilhafte Lösung (Kooperation) wird verhindert aufgrund der Gefahr des einseitigen Defektierens. Dies wird aufgelöst, indem alle Akteure freiwillig Commitment in Form eines sanktionsbehafteten Pfands abgeben (Moral bzw. moralisches Commitment als Produktionsfaktor, vgl. Pies 2008: 33; Will/Hielscher 2014: 226). Diese Lösung eignet sich jedoch nur für eben jene Situationen eines Gefangenendilemmas, in denen eine Kooperation für beide Parteien grundsätzlich vorteilhaft wäre, aber die Gefahr der einseitigen Ausnutzung diese verhindert (z.B. Verzicht auf Rabattschlachten). Nicht bedacht werden dabei etwa Situationen, in denen eine Seite kein Interesse an einer Kooperation hat, also keine gemeinschaftlich vorteilhafte Kooperationslösung existiert.

Diese Einschränkung führt unmittelbar zu der Frage, in welchem Ausmaß in realen Märkten eine Übereinstimmung des Sollens, des Könnens und des Wollens unternehmerischer Verantwortung existiert, wie dies für den Fall eines vollkommenen Marktes festgestellt wurde. Daher beschäftigen wir uns im Folgenden mit den Grenzen sowie generellen Möglichkeiten der Durchsetzung von Normen der CSR. Dabei wird deutlich werden, dass wir aufgrund der Unterschiede in der erfahrungswissenschaftlichen Fundierung im Vergleich zur ökonomischen Ethik und Ordonomik andere Probleme der Durchsetzung von Normen der CSR sehen, so dass sich auch unsere Vorschläge zur institutionellen Absicherung unterscheiden.

\section{Die Durchsetzung von Unternehmensverantwortung im sozialen Kontext}

Ein zentraler Grund für eine fehlende Harmonie zwischen dem Sollen und dem Wollen von CSR unter realen Marktbedingungen wurde bereits genannt: Unter der Annahme der individuellen Nutzenmaximierung kann diese Kongruenz nicht existieren, wenn die verantwortliche Alternative einen geringeren (subjektiven) Nutzen erwarten lässt als die unverantwortliche und wenn die jeweiligen Tauschpartner aufgrund fehlender Ressourcenausstattung nicht in der Lage sind, ihre Zustimmung zu verweigern. Wenn es den 
Akteuren an Ressourcen mangelt, so sind sie unter Umständen dazu gezwungen, für sie negative Tauschverhältnisse einzugehen. Umgekehrt kann ein solcher Mangel an positiver Freiheit Unternehmen daran hindern, sich für die verantwortungsvolle Alternative entscheiden zu können. Aus diesem Grund können institutionelle Rahmenbedingungen, die die positive Freiheit einschränken, sowohl einen Einfluss auf das Wollen als auch das Können von verantwortungsvollem Handeln haben.

Weil sie die Ressourcenausstattung der Akteure explizit berücksichtigt, ergänzen wir die zuvor skizzierte ökonomische Perspektive um die Perspektive der Resource-Dependence-Theorie. Die Aussagekraft dieser Theorie im Kontext der CSR erkannte bereits Freeman (1984: 42, 216), der die Grundgedanken der Theorie nutzte, um erfahrungswissenschaftliche Aussagen darüber zu treffen, warum und wessen Interessen Unternehmen berücksichtigen. Dies wurde in der Folge mehrfach aufgegriffen, jedoch primär als theoretische Basis für den deskriptiven und instrumentellen Stakeholderansatz (vgl. etwa Bondy 2008; Kleinrichert 2008; Lee 2011), weniger aber im Rahmen einer normativen Begründung der Unternehmensverantwortung oder als Grundlage zur Entwicklung von Vorschlägen für eine CSR-konforme Rahmenordnung (vgl. aber Gröneweg/Matiaske 2012). Zu beidem werden wir im Folgenden Aussagen treffen.

Die Resource-Dependence-Theorie von Pfeffer und Salancik (2003) untersucht das Handeln von Organisationen vor dem Hintergrund ihrer sozialen Einbettung in ein marktwirtschaftliches System. Unternehmen werden nicht als autarke Systeme betrachtet, sondern ihr Handeln wird gerade vor dem Hintergrund des jeweiligen sozialen Kontexts erklärt (vgl. Pfeffer/Salancik 2003: 1). Zentrale Annahme ist dabei, dass Unternehmen für ihr Überleben angewiesen sind auf ,kritische“ Ressourcen, die sie von der Umwelt, d.h. verschiedenen Stakeholdern erhalten, da sie nicht alle benötigten Ressourcen selbst kontrollieren. Dabei legt die Theorie einen sehr weiten Ressourcenbegriff zugrunde (vgl. Gröneweg/Matiaske 2012: 6), Organisationen benötigen sowohl materielle (z.B. finanzielle oder physikalische) als auch immaterielle Ressourcen (Informationen, gesellschaftliche Legitimität) (vgl. Pfeffer/Salancik 2003: xii, xvi). Kritische Ressourcen sind dann solche, die für die Organisation überlebensnotwendig sind. Weil das Unternehmen diese jedoch nicht ohne Gegenleistung erhält, ist eine wesentliche Aufgabe von Unternehmen (bzw. deren Leitungen) das „management of demands“ (ebd.: 2). Damit sind für das Unternehmen zwei Probleme verbunden: die Abhängigkeit von anderen Akteuren und die aus dieser Abhängigkeit resultierende Unsicherheit - die wiederum damit zusammenhängt, dass Unternehmen über externe Ressourcen eben keine vollständige Kontrolle ausüben können. Hieraus ziehen Pfeffer und Salancik wiederum zwei Schlüsse: Unternehmen sind erstens in ihren Handlungen stark von der äußeren Umwelt beeinflusst (,external control“), die jedoch nicht so weit geht, einen strukturellen Determinismus zu unterstellen (vgl. Nienhüser 2004: 95). Damit eng verbunden ist zweitens die Annahme, dass Organisationen aktiv versuchen, Unsicherheit und Abhängigkeit zu reduzieren (vgl. ebd.: 102,105), gerade weil dies ihnen dabei hilft, ihre eigenen Ziele besser zu verfolgen (also vor allem Entscheidungen in ihrem Interesse zu beeinflussen). Sie sind hierzu in der Lage, weil Organisationen in der Regel nicht einseitig von anderen Akteuren abhängig sind, sondern sich in einem gegenseitigen Macht-/Abhängigkeitsverhältnis befinden, da beide Akteure Ressourcen kontrollieren, an denen der andere ein Interesse hat. Den Hypothesen der Macht-Abhängigkeits-Theorie von 
Emerson (1962) zufolge, auf die sich die Resource-Dependence-Theorie bezieht (vgl. Pfeffer/Salancik 2003: 27), ist die Abhängigkeit eines Akteurs A umso größer, je mehr Ressourcen B kontrolliert, an denen A ein Interesse hat und umgekehrt, je weniger Ressourcen A kontrolliert, an denen B ein Interesse hat. Darüber hinaus steigt die Abhängigkeit des A, je weniger er die Möglichkeit hat, außerhalb der A-B-Beziehung die benötigte Ressource zu erhalten (vgl. Nienhüser 2004: 93; 2003). Mit anderen Worten: Stakeholder bzw. Lieferanten, die ein Monopol auf eine kritische Ressource besitzen, haben dem Unternehmen gegenüber ein großes Maß an Macht. Darüber hinaus sind Macht-Abhängigkeitsbeziehungen keine statischen sozialen Austauschbeziehungen, sondern dynamisch in der Form, dass Akteure unterschiedliche Machtbalancierungsoperationen vornehmen können, um das Macht-Abhängigkeitsverhältnis zu ihren Gunsten zu verschieben (z.B. Suche nach alternativen Ressourcenquellen, vgl. Nienhüser 2004: 93).

Stellt sich nun die Frage danach, ob Wirtschaftssubjekte unter realen Marktbedingungen verantwortlich in dem Sinne handeln wollen, dass sie die Norm der Wahrung von Handlungsfreiheit respektieren, so zeigt sich bei Gültigkeit der hier unterstellten Prämissen, dass Organisationen zwar ein grundsätzliches Interesse an der eigenen positiven und negativen Handlungsfreiheit haben, nicht jedoch an der Handlungsfreiheit anderer Akteure. Denn den Annahmen der Resource-Dependence-Theorie zufolge steht die eigene Handlungsfreiheit in einem negativen Verhältnis zur Handlungsfreiheit der Stakeholder: Je größer die Macht der Organisation, desto geringer sowohl ihre Abhängigkeit als auch die Unsicherheit - und desto größer die Möglichkeit zur (subjektiven) Maximierung der Nutzenerwartungen. Weil Abhängigkeiten von anderen Akteuren also mit schlechteren Tauschergebnissen einhergehen sowie eine Einschränkung der persönlichen Handlungsfreiheit bedeuten, haben eigennutzorientierte Akteure ein grundsätzliches Interesse daran, Macht auszunutzen und aufzubauen, d.h. die Freiheit anderer nicht zu respektieren oder sogar einzuschränken. Ob Unternehmen verantwortlich handeln, hängt aus dieser Perspektive also nicht davon ab, ob Stakeholder legitime Ansprüche haben, sondern ob sie sie gegenüber dem Unternehmen durchsetzen können. Dies ist den Annahmen der Resource-Dependence-Theorie gemäß nur dann der Fall, wenn folgende Bedingungen erfüllt sind (s. Abb. 1).

1. Das Unternehmen ist sich der Forderung bewusst.

2. Das Unternehmen erhält Ressourcen von diesem Stakeholder.

3. Diese Ressource ist kritisch für das Unternehmen.

4. Der Stakeholder kontrolliert die Allokation, den Zugang oder die Nutzung dieser Ressource, alternative Ressourcenquellen sind nicht verfügbar.

5. Das Unternehmen kontrolliert nicht die Allokation, den Zugang oder die Nutzung von Ressourcen, die für den Stakeholder kritisch sind.

6. Die Handlungen oder Ergebnisse (outputs) des Unternehmens sind sichtbar und können vom Stakeholder bewertet werden hinsichtlich der Frage, ob den Forderungen entsprochen wurde. 
7. Die Erfüllung der Forderungen des Stakeholders widerspricht nicht der Erfüllung von Forderungen anderer Stakeholder.

8. Das Unternehmen kontrolliert nicht das Zustandekommen, die Formulierung und das Erheben der Forderung des Stakeholders.

9. Das Unternehmen ist dazu in der Lage, den Forderungen des Stakeholders zu entsprechen.

10. Die Organisation strebt nach Überleben.

Abbildung 1: Voraussetzungen, unter denen Unternebmen die Forderungen ibrer Stakeholder erfüllen (Quelle: in Anlehnung an Pfeffer/Salancik 2003: 44; Nienbüser 2004: 94)

Gemäß den Handlungshypothesen der Resource-Dependence-Theorie ist zu erwarten, dass Wirtschaftssubjekte ausschließlich die Interessen wichtiger Ressourcenlieferanten in den Blick nehmen werden (vgl. Gröneweg/Matiaske 2012; Thielemann 2008: 218ff.). Insbesondere bei dem Grad der Erfüllung dieser Ansprüche spielt dann auch eine Rolle, ob der entsprechende Lieferant die betreffende Ressource exklusiv kontrolliert (also keine alternativen Ressourcenquellen existieren) sowie welche Ressourcen wiederum das Unternehmen kontrolliert, an denen der Lieferant ein Interesse hat. Anders ausgedrückt: das Macht-/Abhängigkeitsverhältnis spielt eine entscheidende Rolle (Punkte 2 bis 5 sowie $10^{3}$ in Abbildung 1). Ein ressourcenmächtiges Wirtschaftssubjekt ist daher in der Lage, Tauschbedingungen vorzugeben, die das ressourcenabhängige Wirtschaftssubjekt aufgrund seiner fehlenden Ressourcenausstattung annehmen muss, ohne dass seine Interessen (ausreichend) berücksichtigt werden. Damit ist bei ungleicher Ressourcenausstattung nicht zu erwarten, dass Wirtschaftssubjekte ihre Interessen allein aufgrund der Möglichkeit, ihre Zustimmung zu verweigern, vertreten können, sofern es ihnen an der Ausstattung mit kritischen Ressourcen mangelt. Diese fehlende Möglichkeit, die Zustimmung zum Tausch verweigern zu können, steht im Widerspruch zur vorausgesetzten (positiven) Handlungsfreiheit.

Darüber hinaus erkennt die Theorie noch weitere Gründe, warum Unternehmen den Forderungen ihrer Stakeholder nicht nachkommen. Diese liegen entweder darin begründet, dass sie ihnen nicht nachkommen können (siehe unten) oder in Unternehmensstrategien, die darauf abzielen, die eigene Handlungsfreiheit kurz-, mittel- oder langfristig zu erhöhen. So können Unternehmen etwa versuchen, nach alternativen Ressourcenquellen zu suchen, um den Forderungen ihrer Stakeholder mittelfristig zu entgehen (vgl. Nienhüser 2004: 96), z.B. mittels Standortverlagerungen (oder der Drohung hiermit). Oder es werden Unsicherheiten der anderen Marktteilnehmer instrumentalisiert: Häufig ist für Externe nicht unmittelbar erkennbar, ob Unternehmen ihrer Verantwortung tatsächlich nachkommen bzw. die Forderungen der Stakeholder erfüllen (siehe Punkt 6 in Abbildung 1). Dieser Umstand erlaubt es den Unternehmen, kommunikative Legitimitätsfassaden aufzubauen, die nach außen ethisch legitimiertes Handeln sugge- 
rieren (vgl. Delmas/Burbano 2011: 65; Hoßfeld 2011: 29). Darüber hinaus besteht häufig Unsicherheit bezüglich der Frage, wofür Unternehmen konkret verantwortlich gemacht werden sollten, dies ist angesichts komplexer Marktbeziehungen oftmals schwierig zu ermitteln und diskursiv umkämpft. Darum engagieren sich Unternehmen im Rahmen des Lobbying um eine Einflussnahme auf die Normbildung (vgl. Gröneweg/Matiaske 2012: 14; siehe auch Punkt 8 in Abbildung 1) oder verpflichten sich zu (freiwilligen) CSR-Kodizes, in denen Inhalte der Unternehmensverantwortung unklar formuliert sind (zur Kritik an freiwilligen Kodizes vgl. auch Talaulicar 2006).

Weil also Unternehmen diverse Möglichkeiten zur Verfügung stehen, bestehende Marktintransparenzen strategisch zu verwenden, ist es nicht erstaunlich, dass gerade die praktisch angewendete CSR häufig unter der Kritik steht, primär als Vernebelungstaktik genutzt zu werden (vgl. Lee 2011: 293; Sheehy 2015: 626). Unter diesen Voraussetzungen wird dann auch deutlich, warum der Business Case der Ethik nicht (verallgemeinernd) funktionieren kann. Nach dieser Perspektive zahlt sich Ethik aus (vgl. Thielemann 2008; Carroll/Shabana 2010), d.h. es wird unterstellt, dass zumindest langfristig die finanziellen Vorteile eines verantwortungsvollen Handelns jene eines unverantwortlichen Handelns überwiegen. Ein zentraler Aspekt ist hierbei die Gefahr von negativen Sanktionen seitens der Stakeholder, die unverantwortliches Handeln als vergleichsweise kostenineffizient erscheinen lassen: Handeln Unternehmen entgegen den Erwartungen der Stakeholder, so verlieren sie im Extremfall ihre „licence to operate“ (Zinkin 2004, vgl. auch Gröneweg/Matiaske 2012: 9) und damit auch ihre Reputation bzw. die ihnen zugeschriebene Legitimität. Ein in der CSR-Diskussion häufig angeführtes Beispiel ist das von Unternehmensskandalen, die von sogenannten ,watchdogs“ (Medien, NGO) aufgedeckt werden und in deren Konsequenz die Konsumenten das Unternehmen mit Ressourcenentzug abstrafen (vgl. Besiou et al. 2013; Gröneweg/Matiaske 2012: 13). Eine (unterstellte) zunehmende Bedeutung eines solchen gesellschaftlichen Drucks (vgl. hierzu etwa Dawkins/Lewis 2003) legt die Vermutung nah, dass sich auf diesem Weg eine institutionelle Rahmenordnung ,von alleine“ entwickelt, die CSR als Teil einer gewinnmaximierenden Unternehmensstrategie entstehen lässt. Empirisch auftretendes unverantwortliches Handeln wäre damit allein ein Problem der mangelnden Aufklärung, d.h. der begrenzten Rationalität seitens der Unternehmer bzw. Manager. In diesem Zusammenhang verweist Wieland (2007: 71; 2001: 21f.) auch auf die Bedeutung der moralischen Selbstbindung für das Reputationskapital eines Unternehmens.

Allerdings funktioniert dieser implizit unterstellte Mechanismus nur unter bestimmten Bedingungen, die unter den Prämissen der Resource-Dependence-Theorie nicht den Regelfall bilden: Notwendig hierfür ist nämlich zum einen Transparenz bzw. vollständige Information in der Form, dass unternehmerisches Fehlverhalten aufgedeckt wird (als Voraussetzung für dessen Sanktionierung). Zum anderen unterstellt der Business Case implizit, dass das Fehlen von positiver Freiheit kein Problem darstellt bzw. er ignoriert existierende Machtungleichgewichte, die eine Sanktionierung verhindern können. Denn unter realen Marktbedingungen existiert ein Anreiz, (legitime) Ansprüche machtunterlegener Stakeholder nicht zu berücksichtigen, während umgekehrt mächtige Stakeholder dazu in der Lage sind, auch illegitime Ansprüche durchzusetzen (s.u.). In ihrer Heuristik der Dilemmastrukturen ignoriert auch die Ordonomik Situationen, in 
denen eine Transaktionspartei zu einer „Kooperation“ gezwungen wird, weil sie aufgrund fehlender positiver Freiheit diese nicht verweigern kann (z.B. Arbeitnehmer, die aus Angst vor Arbeitsplatzverlust Einbußen bei Entlohnung und Arbeitsbedingungen hinnehmen).

Das hier zugrunde gelegte Verständnis eigennutzorientierten Handelns unterstellt weder, dass alle Akteure opportunistisch handeln (verantwortungsvollem Handeln kann ein intrinsischer Wert zugesprochen werden), noch, dass niemals Eigeninteresse und Gemeinwohl übereinstimmen. Im Gegenteil: Der Business Case lenkt den Blick auf positive Effekte von verantwortungsvollem Handeln auf das Unternehmensimage und die ökonomische Ethik bzw. Ordonomik auf die Existenz von Win-Win-Kooperationslösungen. ${ }^{4}$ Allerdings setzen die Bedingungen auf realen Märkten auch zahlreiche Anreize zu opportunistischem Verhalten. Ethische Probleme, die sich aus dem mangelnden Wollen ergeben, finden sich dann vor allem dort, wo deutliche Machtungleichgewichte und/oder Intransparenzen existieren.

In diesem Zusammenhang stellt sich darüber hinaus die Frage, ob selbst für Akteure, die verantwortungsvoll handeln wollen, strukturell bedingte Grenzen existieren, die verhindern, dass sie verantwortungsvoll handeln können. Solche Grenzen können auf fehlendem Wissen oder fehlenden Mitteln beruhen. Zunächst kann aus ethischer Sicht erstens nur derjenige für seine Taten Verantwortung tragen, der wissentlich (und willentlich) handelt (vgl. Heidbrink 2011: 189). Unter realen Marktbedingungen, d.h. unter der Prämisse genuiner Unsicherheit, ist jedoch keineswegs davon auszugehen, dass sich Unternehmen der legitimen Ansprüche ihrer Stakeholder immer bewusst sind (siehe Punkt 1 in Abbildung 1). Denn in einer komplexen Welt kann weder immer ermittelt werden, welche (kurz- und/oder langfristigen) Effekte unternehmerisches Handeln hat, noch kann das Zurechnungsproblem in allen Fällen gelöst werden (welche Effekte lassen sich welchen Unternehmen zuordnen?).

Zweitens können Unternehmen nur dann verantwortlich handeln, wenn sie faktisch dazu in der Lage sind, den legitimen Anforderungen der Stakeholder zu entsprechen (siehe Punkt 9 in Abbildung 1). Dies ist auch aus ethischer Sicht eine zentrale Frage, denn nur derjenige kann zur Verantwortung gezogen werden, der freiwillig, also nicht unter Zwang handelt (vgl. Heidbrink 2011: 189). Tatsächliche Sachzwänge im Sinne naturgesetzlicher Zwänge schaffen daher „Bereiche, die für moralische Beurteilungen tendenziell unempfänglich sind“ (Kettner 2001: 117). So kann es etwa der Fall sein, dass Unternehmen die Ressourcen nicht zur Verfügung stehen, um den Forderungen der Stakeholder zu entsprechen. Dies ist aber in der Regel kein Problem der Verantwortungsethik, da Unternehmen die Verpflichtung haben, bereits im Vorfeld einer Handlung etwaige Konsequenzen ihres Tuns abzuschätzen. Zwänge, die vom Markt ausgehen, können wiederum keine Sachzwänge sein, denn es sind keine Naturgesetze, die die Geschicke der Marktteilnehmer lenken. Hier liegt ein Kategorienfehler vor, denn der vermeintliche Sachzwang ist ein soziales Phänomen: Reale Märkte sind soziale Systeme; in

\footnotetext{
4

Ähnlich argumentiert auch Wieland (2007: 38, 72), der die Bedeutung der glaubwürdigen Signalisierung von moralischen Intentionen für angestrebte Transaktionen bzw. Kooperationen betont.
} 
diesen existieren keine Naturgesetzmäßigkeiten. Zwänge entstehen hier aus dem Zusammenspiel menschlichen Handelns (vgl. Thielemann 2005; Kettner 2001: 129). Ökonomische Zwänge entstehen dann, wenn moralisches Handeln etwas kostet und auf diese Weise mit komparativen Kostennachteilen verbunden ist. Vor einer solchen „Aufgabe des Profitprinzips" warnen auch Vertreter der Ordonomik, wenn diese mit Wettbewerbsnachteilen verbunden ist (vgl. Will/Hielscher 2014: 226; Pies et al. 2009: 380). Allerdings werden verantwortungsvolle (bzw. kostenineffiziente) Unternehmen nur unter den Bedingungen eines vollkommenen Marktes automatisch vom Markt verdrängt, so dass ihr Handeln gewissermaßen von außen bestimmt wird. Jenseits eines vollkommenen Marktes können Unternehmen in der Regel „Ineffizienzen“ innerhalb gewisser Grenzen aushalten (vgl. auch Ulrich 2008: 159-175).

Außerdem haben Unternehmen grundsätzlich immer eine Wahl: Sie können mindestens immer zwischen dem unverantwortlichen Handeln und der Liquidation des Unternehmens entscheiden. Zwar tragen aus philosophischer Sicht Unternehmen auch in solchen Extremsituationen Verantwortung für ihr Handeln - entweder man trägt für eine Handlung Verantwortung oder nicht (vgl. Aßländer 2010: 194) -, dennoch wäre ihre moralische Bewertung eine andere: „Moralische Verantwortung setzt das Vorhandensein entsprechender Freiräume und Handlungsspielräume voraus, das heißt das faktische Vermögen, die zugeschriebene Verantwortung auch übernehmen zu können“ (vgl. Kleinfeld/Henze 2010: 60). Anders ausgedrückt: Das Ausmaß an unternehmerischer Verantwortung korreliert negativ mit dem Ausmaß an positiver und negativer Handlungsfreiheit des Unternehmens.

Und in diesem Sinne existieren neben den oben skizzierten ökonomischen Zwängen auch weitere strukturelle Zwänge, die vom Markt ausgehen - bzw. von dem Umstand, dass Unternehmen aufgrund der ressourcenbedingten Verflechtungen mit anderen Wirtschaftssubjekten zahlreichen Abhängigkeiten unterliegen. Gemäß der ResourceDependence-Theorie geraten Unternehmen in ein moralisches Dilemma, wenn Forderungen anderer Stakeholder der Verantwortungsübernahme im Wege stehen (siehe Punkt 7 in Abbildung 1). In solchen Fällen kann verantwortliches Handeln dazu führen, dass Stakeholder dem Unternehmen kritische Ressourcen nicht (mehr) zur Verfügung stellen, etwa weil dieses den Erwartungen an ein ökonomisch rationales Unternehmen nicht (mehr) entspricht (vgl. Walgenbach/Meyer 2008). Dies ist regelmäßig dann der Fall, wenn von mächtigen Stakeholdern ein normativer Druck ${ }^{5}$ ausgeht hinsichtlich der Erfüllung bestimmter ökonomischer und nicht-ökonomischer Ziele (z.B. kurzfristige/langfristige Gewinne, Standorterhaltung, geringe Preise) oder der Durchführung bestimmter Mittel, die diese Ziele vermeintlich erreichen helfen (z.B. Downsizing, vgl. Hoßfeld 2011: 32f.). Hier sind es dann mächtige Stakeholder, die in Kauf nehmen (bzw. fordern), dass das Unternehmen ihre Ansprüche auch auf eine Art erfüllt, die mit der gezielten Beschneidung der Handlungsfreiheit anderer, schwächerer Stakeholder einhergeht. Strukturelle Zwänge lassen sich also nicht auf eindeutig konturierte (Gefange-

$5 \quad$ Auch Wieland (2007: 46) spricht von der „Erzwingungsmacht“ von NGOs, ohne jedoch diese Machtverhältnisse genauer zu spezifizieren. 
nen-)Dilemmasituationen herunterbrechen, sondern gewinnen an Komplexität aufgrund der zahlreichen unterschiedlichen - auch: wahrgenommenen - Erwartungshaltungen und Abhängigkeitsbeziehungen.

Damit zeigen sich nicht nur in der Motivation, sondern auch in der Gelegenheit deutliche Grenzen der Realisier- bzw. Durchsetzbarkeit von CSR-Normen: Unter der Voraussetzung, dass Unternehmensverantwortung die Wahrung von Handlungsfreiheit bedeutet, sowie unter den Prämissen der genuinen Unsicherheit und der Existenz von Machtasymmetrien stimmen unter realen Marktbedingungen Wollen, Können und Sollen verantwortungsvollen Handelns nicht überein. Weder ergibt sich eine solche Kongruenz unmittelbar aus dem Marktmechanismus noch existieren in realen Märkten (dezentrale) Entwicklungsbedingungen und Mechanismen, die eine institutionelle Rahmenordnung schaffen, die Unternehmen generell für verantwortungsvolles Handeln belohnt und unverantwortliches Handeln bestraft.

Angesichts strukturell bedingter Zwänge und „Verlockungen“, die unverantwortliches Handeln aus eigennutzorientierter Sicht attraktiv erscheinen lassen, ist es daher Aufgabe der Marktordnung, eine ,institutionell gesicherte Arena für freiwilligen Tausch und freiwillige Kooperation bereitzustellen" (Vanberg 2001; 2006: 45), d.h. Möglichkeiten für die Durchsetzung von CSR-Normen zu schaffen. Aus der hier skizzierten Perspektive der Resource-Dependence-Theorie existieren zwei zentrale Mechanismen, die dazu beitragen können, dass Unternehmen (legitime) Ansprüche ihrer Stakeholder berücksichtigen: Transparenz bezüglich unternehmerischen Handelns und der Inhalte unternehmerischer Verantwortung (insbesondere die Frage, wann negative und positive Freiheit gewährt sind) sowie das Ausbleiben deutlicher Macbtasymmetrien, die die positive Freiheit machtunterlegener Transaktionspartner gefährden. Aufgabe einer institutionellen Rahmenordnung ist es daher, Mechanismen zu schaffen, die sowohl eine inhaltliche Konkretisierung von unternehmerischer Verantwortung als auch deren Durchsetzung sicherstellt. Für eine solche institutionelle Rahmenordnung existieren verschiedene Ansatzpunkte. Dabei ist grundsätzlich zu unterscheiden zwischen einem institutionellen Rahmen für generelle Regeln sowie einem Rahmen für spezielle, d.h. einzelfallbezogene Regeln. Generelle Regeln kommen üblicherweise dort zum Einsatz, wo es möglich und erwünscht ist, übergreifende Regeln zu schaffen, die unabhängig von konkreten Tauschbeziehungen sind und einen allgemeingültigen Charakter haben. Sie sind nur da möglich, wo Verantwortlichkeiten eindeutig zu klären und langfristig gültig sind. Sie geben den Wirtschaftssubjekten Regelsicherheit und werden primär dazu eingesetzt, jene mit einer schlechten Ressourcenausstattung zu schützen bzw. zu verhindern, dass jene mit einer guten Ressourcenausstattung ihre Freiheit missbrauchen können (siehe auch die Funktionen einer Rechtsordnung, vgl. etwa Isensee 1983; Heinrich 2000). Beispiele hierfür sind etwa Mindestnormen (z.B. bzgl. der Arbeitsbedingungen) oder Transparenzregeln, die es Unternehmen erschweren, Greenwashing zu betreiben. Generelle Regeln können staatlichen Ursprungs sein und vom Gesetzgeber abgesichert werden, sie müssen es jedoch nicht. Gerade in Bereichen, in denen einzelstaatliche Lösungen nicht greifen, bieten sich Regelungen an, die unabhängig von einzelnen Staaten sind. Tatsächlich beschäftigt sich ein Großteil der praktischen und wissenschaftlichen CSR-Diskussion mit außergesetzlichen Regelwerken, die von Politik, NGOs und Un- 
ternehmen gemeinsam entwickelt werden. So bieten etwa die OECD-Leitsätze für multinationale Unternehmen deutliche inhaltliche Anknüpfungspunkte an die hier zugrunde gelegte Perspektive, denn unter anderem werden hier mehr Transparenz und der Schutz insbesondere wenig mächtiger Stakeholder (Arbeitnehmer, Verbraucher) gefordert (vgl. OECD 2011). Also ließe sich argumentieren, dass die real praktizierte CSR bereits dem hier vorgestellten Konzept einer erfahrungswissenschaftlich begründeten CSR sehr nahe kommt. Allerdings weist diese Leitlinie einen entscheidenden Nachteil auf: die Freiwilligkeit. Es ist wenig sinnvoll, CSR als ein rein freiwilliges Konzept zu verstehen. Dies ist weder aus ethischer Perspektive vernünftig - es ist allenfalls „möglich, sich seiner Verantwortung zu stellen oder sich ihr zu entziehen“ (Aßländer 2010: 194) - noch kann vor dem Hintergrund der hier skizzierten erfahrungswissenschaftlichen Erkenntnissen davon ausgegangen werden, dass Unternehmen ihrer Verantwortung auch immer nachkommen. Um eine Wirksamkeit dieser Normen zu gewährleisten, sind daher immer Sanktionsmechanismen notwendig, die ein Befolgen dieser Regeln relativ vorteilhaft erscheinen lässt (vgl. Gröneweg/Matiaske 2012), z.B. Konventionalstrafen.

Jedoch lässt sich nicht alles (sinnvoll) mittels verallgemeinerbaren, langfristig gültigen Regeln und damit einhergehenden institutionellen Sanktionsmechanismen regulieren. Zentrales Charakteristikum einer Marktordnung sind Tauschbeziehungen zwischen Transaktionspartnern in Form von Verhandlungen. Hierbei werden von den betroffenen Tauschpartnern bzw. deren Repräsentanten auch spezielle Regeln ausgehandelt, die die Verantwortungsübernahme aller beteiligten Parteien betreffen. Aufgrund von - zumeist strukturell bedingten - Machtasymmetrien ist es hier jedoch notwendig, institutionelle Mechanismen zu schaffen, die die positive Handlungsfreiheit der machtunterlegenen Partei sicherstellt bzw. erhöht. Ein Beispiel hierfür ist etwa die Arbeitnehmermitbestimmung, in deren Rahmen die Arbeitsbedingungen zwischen Arbeitgeber- und Arbeitnehmerseite auf betrieblicher und tariflicher Ebene ausgehandelt werden. Weil sich Arbeitsbedingungen in einem fortlaufenden Entwicklungsprozess befinden, überlässt es der Staat - mit Ausnahme der Formulierung von Mindeststandards - den beteiligten Parteien, diese zu regulieren (dies gilt insbesondere im Rahmen der Tarifautonomie, vgl. Müller-Jentsch 1997: 48f.). Weil aber bei solchen Verhandlungslösungen in der Regel starke Machtungleichgewichte zuungunsten der Arbeitnehmerseite existieren, werden diese Verhandlungen institutionell abgesichert. Dies erfolgt zum einen durch die Ressourcenbündelung der Arbeitnehmer (Gründung von Betriebsräten und Gewerkschaften, vgl. Nienhüser 2014) und zum anderen durch Mitbestimmungsgesetze, deren kodifizierten, staatlich zugesicherten Rechte für die Arbeitnehmer Ressourcen bilden, die ihre positive Handlungsfreiheit gegenüber der Arbeitgeberseite erhöht. Auf diese Weise greift der Staat nicht inhaltlich, sondern lediglich prozedural in die Transaktionsbeziehungen von Wirtschaftssubjekten ein.

Ähnlich wie ökonomische Ethik und Ordonomik unterscheiden wir also zwei grundlegende Mittel, mit denen CSR-Normen realisiert werden können: Staatliche Regulierung und Verhandlungslösungen (vgl. etwa Beckmann 2010). Jedoch wollen die genannten Ansätze solche Normen ausschließlich mit finanziellen Anreizen durchsetzen: Verbote, Gebote und Subventionen einerseits, Kooperationslösungen aus Gefangenendilemmata andererseits. Der hier vorgestellte Ansatz wehrt sich jedoch gegen eine systematische 
Überschätzung ökonomischer Anreize, auch weil Unternehmen nicht allein ökonomische Ziele verfolgen (und unklar ist bzw. variieren kann, welche ökonomischen Ziele sie verfolgen). Die Resource-Dependence-Theorie öffnet den Blick auf Lösungen, bei denen nicht unmittelbar bei der unternehmerischen Anreizgestaltung angesetzt wird, sondern bei dem Machtverhältnis zwischen dem einzelnen Unternehmen und seinen Stakeholdern. Dies kann einerseits durch eine Stärkung machtunterlegener Stakeholder erfolgen, etwa durch die Schaffung prozeduraler Regeln, die Machtverhältnisse angleichen (z.B. Mitbestimmungsgesetze) oder durch die (auch finanzielle) Förderung von NGOs. Andererseits kann dies durch die Schwächung machtüberlegener Akteure erfolgen, z.B. durch Transparenzregeln oder Regeln für Lobbyismus. Dabei bleibt die Gestaltung einer institutionellen Rahmenordnung - sowohl bezüglich genereller und spezieller als auch inhaltlicher und prozeduraler Regeln - eine komplexe Aufgabe. Insofern ist der Ordonomik zuzustimmen, dass Wirtschaftssubjekte auch eine Mitverantwortung an der Gestaltung des Systems tragen (sog. Diskurs- bzw. Aufklärungsverantwortung, vgl. Pies et al. 2009: 386; Beckmann 2010). Aber auch eine solche „Ordnungspolitik zweiter Ordnung" (Pies 2012: 237) lässt sich nicht allein unter Rückgriff auf Überlegungen zu gegenseitig vorteilhaften Kooperationslösungen (vgl. hierzu etwa Beckmann 2010: 245250) gestalten - und bleibt ebenso immer lückenhaft, so dass auch hier stets die Notwendigkeit für Verantwortung auf der Handlungsebene der Akteure erhalten bleibt.

\section{5. $\quad$ Fazit}

Gegenstand unseres Beitrags ist die Erarbeitung eines ethisch und erfahrungswissenschaftlich fundierten Konzepts der CSR in einer Marktwirtschaftsordnung. Das ethische Fundament des von uns entwickelten CSR-Konzepts bildet Freiheit als übergreifendes gesellschaftliches Ziel demokratischer Verfassungen. Das erfahrungswissenschaftliche Fundament sind Handlungshypothesen und ein Marktverständnis unter genuiner Unsicherheit sowie mit dem Resource-Dependence-Ansatz eine Unternehmenstheorie unter Berücksichtigung von Macht. Gerade dieses erfahrungswissenschaftliche Fundament unterscheidet unseren Ansatz von diskursethisch begründeten Verantwortungskonzepten, vom Business Case, sowie von Verantwortungskonzepten der ökonomischen Ethik und Ordonomik. Das von uns vorgelegte Konzept liefert die „Gebrauchsanweisung“ für die Erarbeitung einer ethisch und erfahrungswissenschaftlich fundierten Tauschordnung (nicht die Tauschordnung selbst) und ist folgendermaßen charakterisiert:

Ausgehend von Freiheit als Ziel eines marktwirtschaftlichen Systems ist Verantwortung von Unternehmen nicht auf Gewinnmaximierung reduziert. Vielmehr sollen Unternehmen verpflichtend (und nicht etwa freiwillig) als moralische Grenze eigennutzorientierten Handelns die positive und negative Handlungsfreiheit anderer Marktteilnehmer wahren. Wann Handlungsfreiheit gewahrt wird, ist durch Rückgriff auf ethische Konzeptionen zur Handlungsfreiheit problembezogen zu konkretisieren. Unternehmensverantwortung ist hierauf beschränkt, Unternehmen sind weder inhaltlich auf einen bestimmten Unternehmenszweck festgelegt noch tragen sie Verantwortung für karitative Tätigkeiten.

Unter Berücksichtigung der Hypothesen der Resource-Dependence-Theorie ist nicht davon auszugehen, dass Unternehmen immer in diesem Sinne verantwortlich handeln wollen. Unternehmen versuchen Macht aufzubauen, um Unsicherheit und Abhängigkeit 
zu reduzieren und ihre Handlungsfreiheit zu vergrößern. Demzufolge ist zu erwarten, dass sie die Ansprüche mächtiger Stakeholder erfüllen, aber auch mithilfe von Legitimitätsfassaden verantwortliches Handeln vorgeben sowie über Lobbying versuchen werden, Einfluss auf inhaltliche Konkretisierungen von Verantwortung zu nehmen. Auch wenn Unternehmen (durchaus eigennutzorientiert) verantwortungsvoll handeln wollen, ist nicht unbedingt gewährleistet, dass sie dies können. Denn es ist möglich, dass sie selbst von Stakeholdern abhängig sind, die andere Erwartungen, z.B. Gewinnmaximierung auch unter Inkaufnahme der Einschränkung der Handlungsfreiheit von Marktteilnehmern, an sie stellen. Zwar existieren auf realen Märkten keine naturgesetzlichen Zwänge, aber durchaus strukturellen Zwänge.

Dieser Durchsetzungsproblematik muss die institutionelle Rahmenordnung Rechnung tragen. Diese beschränkt sich nicht auf gesetzliche Regelungen, sondern umfasst auch außergesetzliche Regelungen, die besonders im globalen Kontext erforderlich werden. Sowohl für gesetzliche als auch außergesetzliche Regeln gilt, dass Verantwortung im hier verstandenen Sinne (Wahrung positiver und negativer Handlungsfreiheit) inhaltlich zu konkretisieren ist, dass Regeln erforderlich sind, die das Handeln von Unternehmen transparent machen und nicht verantwortliches Handeln sanktionieren. Dabei sollten problembezogen sowohl generelle Regeln als auch spezielle Regeln in Form von Verhandlungslösungen eingesetzt werden. Bei letzteren sind allerdings in besonderem Maße Machtasymmetrien in den Blick zu nehmen, und Machtungleichgewichten ist entgegenzuwirken. Unter genuiner Unsicherheit lässt sich nicht ausschließen, dass Unternehmen nicht so verantwortlich handeln, wie sie sollen oder so verantwortlich handeln können, wie sie sollen (und wollen). Unserer Auffassung nach sprechen aber gute Gründe dafür, dass die nach dem von uns vorgelegten Konzept entwickelte Tauschordnung diese Fälle verringert.

\section{Literatur}

Aaken, D. van (2012): Individuelle Freiheit als Grundlage normativer Ökonomik, in: Zeitschrift für Betriebswirtschaft, Vol. 82/No. 6, 81-102.

Agninis, H./Glavas, A. (2012): What We Know and Don't Know About Corporate Social Responsibility: A Review and Research Agenda, in: Journal of Management, Vol. 38/No. 4, 932-968.

Albert, H. (1985): Treatise on Critical Reason, Princeton: University Press.

Albert, H. (2000): Kritischer Rationalismus, Tübingen: Mohr Siebeck.

Aßländer, M. S. (2010): Die Normierung des Guten. Gesellschaftliches Engagement auf dem Prüfstand, in: Braun, S. (Hrsg.): Gesellschaftliches Engagement von Unternehmen. Der deutsche Weg im internationalen Kontext, Wiesbaden: VS Verlag für Sozialwissenschaften, 187-201.

Aßländer, M. S./Löhr, A. (2010): Einführung: Zum Klärungsbedarf der Modevokabel „Corporate Social Responsibility“, in: Aßländer, M. S./Löhr, A. (Hrsg.): Corporate Social Responsibility in der Wirtschaftskrise. Reichweiten der Verantwortung, München, Mering: Hampp, 11-32.

Beckmann, M. (2010): Ordnungsverantwortung: Rational Choice als ordonomisches Forschungsprogramm, Berlin: Wissenschaftlicher Verlag Berlin. 
Besiou, M./Hunter, M. L./Wassenhove, L. N. van (2013): A Web of Watchdogs: Stakeholder Media Networks and Agenda-setting in Response to Corporate Initiatives, in: Journal of Business Ethics, Vol. 118/No. 4, 709-729.

Boettcher, E. (1974): Kooperation und Demokratie in der Wirtschaft, Tübingen: Mohr.

Bondy, K. (2008): The Paradox of Power in CSR: a Case Study on Implementation, in: Journal of Business Ethics, Vol. 82/No. 2, 307-323.

Brennan, G./Buchanan, J. M. (1985): The Reason of Rules, Cambridge: Cambridge University Press.

Buchanan, J. M./Vanberg, V. J. (1991): The Market as a Creative Process, in: Economics and Philosophy, Vol. 7/No. 2, 167-186.

Burkatəki, E./Löhr, A. (2008): Wirtschaftskriminalität und Ethik - einführende Anmerkungen, in: Löhr, A./Burkatzki, E. (Hrsg.): Wirtschaftskriminalität und Ethik, München, Mering: Hampp, 11-21.

Carroll, A. B. (1991): The Pyramid of Corporate Social Responsibility: Toward the Moral Management of Organizational Stakeholders, in: Business Horizons, Vol. 34/No. 4, 39-48.

Carroll, A. B. (1999): Corporate Social Responsibility - Evolution of a Definitional Construction, in: Business and Society, Vol. 38/No. 3, 268-295.

Carroll, A. B. (2008): A History of Corporate Social Responsibility: Concepts and Practices, in: Crane, A./McWilliams, A./Matten, D./Moon, J./Siegel, D. (Eds.): The Oxford Handbook of Corporate Social Responsibility, Oxford: Oxford University Press 19-46.

Carroll, A. B./Shabana, K. M. (2010): The Business Case for Corporate Social Responsibility: a Review of Concepts, Research and Practice, in: International Journal of Management Reviews, Vol. 12/No. 1, 85-105.

Crane, A./McWilliams, A./Matten, D./Moon, J./Siegel, D. (2008): Conclusion, in: Crane, A./McWilliams, A./Matten, D./Moon, J./Siegel, D. (Eds.): The Oxford Handbook of Corporate Social Responsibility, Oxford: Oxford University Press, 568-576.

Dahlsrud, A. (2008): How Corporate Social Responsibility is Defined: an Analysis of 37 Definitions, in: Corporate Social Responsibility and Environmental Management, Vol. 15/No. 1, 1-13.

Dawkins, J./Lewis, S. (2003): CSR in Stakeholder Expectations and Their Implication for Company Strategy, in: Journal of Business Ethics, Vol. 44/No., 185-193.

Delmas, M. A./Burbano, V. C. (2011): The Drivers of Greenwashing, in: California Management Review, Vol. 54, No. 1, 64-87.

Devinney, T. M. (2009): Is the Socially Responsible Corporation a Myth? The Good, the Bad, and the Ugly of Corporate Social Responsibility, in: Academy of Management Perspectives, Vol. 23, No. 2, 44-56.

Donaldson, T./Preston, L. E. (1995): The Stakeholder Theory of the Corporation: Concepts, Evidence, and Implications, in: Academy of Management Review, Vol. 20/No. 1, 65-91.

Emerson, R. M. (1962): Power-Dependence Relations, in: American Sociological Review, Vol. 27/No. 1, 31-41.

Europäische Kommission (2001): Grünbuch. Europäische Rahmenbedingungen für die soziale Verantwortung der Unternehmen, Luxemburg: Amt für amtliche Veröffentlichungen der Europäischen Gemeinschaften. 
Europäische Kommission (2011): Mitteilung der Kommission an das Europäische Parlament, den Rat, den Europäischen Wirtschafts- und Sozialausschuss und Ausschuss der Regionen. Grundstoffmärkte und Rohstoffe: Herausforderungen und Lösungsansätze, Brüssel: Europäische Kommission.

Evan, W. M./Freeman, R. E. (1988): A Stakeholder Theory of the Modern Corporation: Kantian Capitalism, in: Beauchamp, T. L./Bowie, N. E. (Eds.): Ethical Theory and Business, Englewood Cliffs: Prentice Hall, 97-106.

Falk, J. (2012): Freiheit als politisches Ziel. Grundmodelle liberalen Denkens bei Kant, Hayek und Böckenförd, Frankfurt a.M.: Campus.

Freeman, R. E. (1984): Strategic Management: A Stakeholder Approach, Boston, MA: Pitman.

Freeman, R. E./Pbillips, R. A. (2002): Stakeholder Theory: A Libertarian Defense, in: Business Ethics Quarterly, Vol. 12/No. 3, 331-349.

Friedman, M. (1970): The Social Responsibility of Business is to Increase its Profits, in: New York Times Magazine, September 13, 32-33.

Gadenne, V. (2002): Hat der kritische Rationalismus noch etwas zu lehren?, in: Böhm, J. M./Holweg, H./Hoock, C. (Hrsg.): Karl Poppers kritischer Rationalismus heute, Tübingen: Mohr Siebeck, 58-78

Gröneweg, C./Matiaske, W. (2012): Gullivers Fesseln - Corporate Social Responsibility als Normbildung, Bericht Nr. 25, Werkstatt für Organisation und Personalforschung e. V. Berlin.

Heidbrink, L. (2011): Der Verantwortungsbegriff der Wirtschaftsethik, in: Aßländer, M. (Hrsg.): Handbuch Wirtschaftsethik, Stuttgart: Metzler, 188-197.

Heinrich, C. (2000): Formale Freiheit und materiale Gerechtigkeit, Tübingen: Mohr.

Homann, K. (2002): Wettbewerb und Moral, in: Lütge, C. (Hrsg.): Vorteile und Anreize, Tübingen: Mohr Siebeck, S. 23-44.

Homann, K. (2014): Sollen und Können, Wien: Ibera Verlag.

Homann, K./Blome-Drees, F. (1992): Wirtschafts- und Unternehmensethik, Göttingen: Vandenhoeck \& Ruprecht.

Homann, K./Lütge, C. (2013): Einführung in die Wirtschaftsethik, 3. Aufl., Berlin: LIT Verlag.

Homann, K./Suchanek, A. (2005): Ökonomik: Eine Einführung, 2. Aufl., Tübingen: Mohr Siebeck.

Hoßfeld, H. (2011): Sprachliche Legitimation von Personalabbaumaßnahmen. Eine Argumentations- und Metaphernanalyse der Massenkommunikation zweier Großbanken, München, Mering: Hampp.

Isensee, J. (1983): Das Grundrecht auf Sicherheit: Zu den Schutzpflichten des freiheitlichen Verfassungsstaates, Berlin, New York: de Gruyter.

Kettner, M. (2001): Sachzwang - Über einen kritischen Grundbegriff der Wirtschaftsethik, in: Koslowski, P. (Hrsg.): Wirtschaftsethik - Wo ist die Philosophie?, Heidelberg: PhysicaVerlag, 117-144.

Kleinewefers, H. (2008): Einführung in die Wohlfahrtsökonomie, Stuttgart: Kohlhammer.

Kleinfeld, A./Henze, B. (2010): Wenn der Maßstab fehlt - oder wann ist CSR (unternehmens)ethisch vertretbar?, in: Aßländer, M. S./Löhr, A. (Hrsg.): Corporate Social Responsibility in der Wirtschaftskrise. Reichweiten der Verantwortung. München, Mering: Hampp, 49-71. 
Kleinrichert, D. (2008): Ethics, Power and Communities: Corporate Social Responsibility Revisited, in: Journal of Business Ethics, Vol. 78/No. 3, 475-485.

Kurucz, E./Colbert, B./Wheeler, D. (2008): The Business Case for Corporate Social Responsibility, in: Crane, A./McWilliams, A./Matten, D./Moon, J./Siegel, D. (Eds.): The Oxford Handbook of Corporate Social Responsibility, Oxford: Oxford University Press, 83112.

Ladewig, B. (2011): Freiheit, in: Göhler, G./Iser, M./Kerner, I. (Hrsg.): Politische Theorie, 2. Aufl., Wiesbaden: VS-Verlag, 79-93.

Lee, M.-D. P. (2011): Configuration of External Influences: the Combined Effects of Institutions and Stakeholders on Corporate Social Responsibility Strategies, in: Journal of Business Ethics, Vol. 102/No. 2, 281-298.

Lin-Hi, N. (2009): Eine Theorie der Unternehmensverantwortung, Berlin: Erich Schmidt Verlag. Loew, T./ Ankele, K./Braun, S./Clausen, J. (2004): Bedeutung der internationalen CSR-Diskussion für Nachhaltigkeit und die sich daraus ergebenden Anforderungen an Unternehmen mit Fokus Berichterstattung. Endbericht. Münster und Berlin. Link: http://wcyukbf.upj.de/fileadmin/user_upload/MAIN-dateien/Themen/Einfuehrung/ioew_csr_diskussion_2004.pdf (zuletzt abgerufen am 30.10.2014).

Müller-Jentsch, W. (1997): Soziologie der Industriellen Beziehungen. Eine Einführung, 2., überarb. und erw. Aufl., Frankfurt a.M.: Campus.

Nida-Rümelin, J. (2011): Verantwortung, Stuttgart: Reclam.

Nienbüser, W. (2003): Macht, in: Martin, A. (Hrsg.): Organizational Behavior - Verhalten in Organisationen, Stuttgart: Kohlhammer, 139-172.

Nienbüser, W. (2004): Die Resource Dependence-Theorie - wie (gut) erklärt sie Unternehmensverhalten?, in: Festing, M./Martin, A./Mayrhofer, W./Nienhüser, W. (Hrsg.): Personaltheorie als Theorie der Unternehmung. Festschrift zum 65. Geburtstag von Prof. Dr. Wolfgang Weber, München, Mering: Hampp, 87-119.

Nienbüser, W. (2014): Arbeit - Macht - Politik: Machtverschiebungen in den Arbeitsbeziehungen, deren Folgen und was man tun kann, in: Vedder, G./Pieck, N./Schlichting, B./Schubert, A. (Hrsg.): Befristete Beziehungen, München, Mering: Hampp, 233-252.

Nutzinger, H. G. (1992): Der Begriff Verantwortung aus ökonomischer und sozialethischer Sicht, in: Homann, K. (Hrsg.): Aktuelle Probleme der Wirtschaftsethik, Berlin: Duncker \& Humboldt, 43-67.

OECD (2011): Die OECD-Leitsätze für multinationale Unternehmen. Neufassung 2011, Paris: OECD.

Ortmann, G. (2009): Management in der Hypermoderne. Kontingenz und Entscheidung, Wiesbaden: VS Verlag für Sozialwissenschaften.

Peter, F. (2004): Demokratische Legitimation von Marktarrangements: Auf dem Weg zu einer Wirtschaftsethik als politische Ethik, in: Zeitschrift für Wirtschafts- und Unternehmensethik, Jg. 5/Heft 2, 163-175.

Pies, I. (2008): Markt und Organisation: Programmatische Überlegungen zur Wirtschafts- und Unternehmensethik, in: Kersting, W. (Hrsg.): Moral und Kapital. Grundfragen der Wirtschafts- und Unternehmensethik, Paderborn: Mentis, 27-58. 
Pies, I. (2012): Systemverantwortung versus Ordnungsverantwortung? - Eine ordonomische Replik auf den Beitrag von Ludger Heidbrink, in: ORDO - Jahrbuch für die Ordnung von Wirtschaft und Gesellschaft, Jg. 63, 233-240.

Pies, I./Hielscher, S. (2008): Der systematische Ort der Zivilgesellschaft. Welche Rolle weist eine ökonomische Theorie der Moral zivilgesellschaftlichen Organisationen in der modernen Gesellschaft zu?, in: Zeitschrift für Wirtschafts- und Unternehmensethik, Jg. 9/Heft 3, 337-344.

Pies, I./Hielscher, S. (2014): Verhaltensökonomik versus Ordnungsethik, in: Zeitschrift für Wirtschafts- und Unternehmensethik, Jg. 15/Heft 3, 398-420.

Pies, I./ Hielscher, S./Beckmann, M. (2009): Moral Commitments and the Societal Role of Business: An Ordonomic Approach to Corporate Citizenship, in: Business Ethics Quarterly, Vol. 19/No. 3, 375-401.

Pfeffer, J./Salancik, G. R. (2003): The External Control of Organizations: a Resource Dependence Perspective, Stanford, California: Stanford Business Books.

Richter, R./Furubotn, E. G. (2010): Neue Institutionenökonomik, 4. Aufl., Tübingen: Mohr.

Schlecht, O. (1990): Grundlagen und Perspektiven der sozialen Marktwirtschaft, Tübingen: Mohr.

Schmidt, R. H./Terberger, E. (1997): Grundzüge der Investitions- und Finanzierungstheorie, 4. Aufl., Wiesbaden: Gabler.

Schmiel, U./Hoßfeld, H. (2014): Gewinnmaximierung als marktwirtschaftlich legitimes unternehmerisches Ziel? - Zur Konkretisierung marktwirtschaftlich legitimer Gewinnverfolgung am Beispiel von Steuerausweichgestaltungen, in: Jahrbuch Ökonomie und Gesellschaft, Band 26, 291-318.

Schneider, D. (1987): Allgemeine Betriebswirtschaftslehre, 3. Aufl., München: Oldenbourg.

Schreck, P. (2012): Der Business Case for Corporate Social Responsibility, in: Schneider, A./Schmidpeter, R. (Hrsg.): Corporate Social Responsibility. Verantwortungsvolle Unternehmensführung in Theorie und Praxis, Berlin, Heidelberg: Springer-Gabler, 67-86.

Sen, A. (1988): Freedom of Choice, in: European Economic Review, Vol. 32/No. 2-3, 269-294.

Sheehy, B. (2015) „Defining CSR: Problems and Solutions“, Journal of Business Ethics, Vol. 131/No. 3, 625-648.

Suchanek, A. (2007): Ökonomische Ethik, 2. Aufl., Tübingen: Mohr Siebeck.

Streit, M. E. (1988): Freiheit und Gerechtigkeit. Ordnungspolitische Aspekte zweier gesellschaftlicher Grundwerte, in: ORDO: Jahrbuch für die Ordnung von Wirtschaft und Gesellschaft, Jg. 39/Heft 1, 33-53.

Talaulicar, T. (2006): Unternehmenskodizes. Typen und Normierungsstrategien zur Implementierung von Unternehmensethik, Wiesbaden: Deutscher Universitäts-Verlag.

Thielemann, U. (2005): Freiheit unter den Bedingungen des Marktes. Oder doch gegenüber der Marktlogik? Vom verfehlten Umgang mit Sachzwängen, in: Schmidinger, H./Sedmak, C. (Hrsg.): Der Mensch - ein freies Wesen?, Darmstadt: WBG, 261-288.

Thielemann, U. (2008): Unternehmensverantwortung ethisch ernst genommen: The Case against the Business Case und die Idee verdienter Reputation, in: Heidbrink, L./Hirsch, A. (Hrsg.): Verantwortung als marktwirtschaftliches Prinzip, Frankfurt a.M.: Campus, 199-230.

Ulrich, P. (2008): Integrative Wirtschaftsethik, Grundlagen einer lebensdienlichen Ökonomie, 4. Aufl., Bern: Haupt-Verlag. 
Vanberg, V. (1997): Die normativen Grundlagen von Ordnungspolitik, in: ORDO Jahrbuch für die Ordnung von Wirtschaft und Gesellschaft, Jg. 48, 707-726.

Vanberg, V. (2001): Konstitutionenökonomische Überlegungen zum Konzept der Wettbewerbsfreiheit, in: ORDO Jahrbuch für die Ordnung von Wirtschaft und Gesellschaft, Jg. 52, $37-62$.

Vanberg, V. (2003), Evolutorische Ökonomik: Homo Oeconomicus, Markt und Institutionen, in: Evolution in den Natur-, Sozial- Geisteswissenschaften, Bern: Haupt-Verlag, 117137.

Vanberg, V. (2005): Rationalitätsprinzip und Rationalitätshypothesen: Zum methodologischen Status der Theorie rationalen Handelns, in: Siegenthaler, H. (Hrsg.): Rationalität im Prozess kultureller Evolution, Rationalitätsunterstellungen als eine Bedingung der Möglichkeit substantieller Rationalität des Handelns, Tübingen: Mohr Siebeck, 33-63.

Vanberg, V. (2006): Marktwirtschaft und Gerechtigkeit - Zu F. A. Hayeks Kritik am Konzept der ,sozialen Gerechtigkeit“, in: Held, M./Kubon-Gilke, G./Sturn, R. (Hrsg.): Soziale Sicherung in Marktgesellschaften. Normative und institutionelle Grundfragen der Ökonomik, Jahrbuch 5, Marburg: Metropolis, 39-69.

Vanberg, V. (2007): Corporate Social Responsibility and the ,Game of Catallaxy': the Perspective of Constitutional Economics, in: Constitutional Political Economy, Vol. 18/No 18, 199-222.

Varian, H. R. (2011): Grundzüge der Mikroökonomik, 8. Aufl., München: Oldenbourg.

Walgenbach P./Meyer, R. (2008): Neoinstitutionalistische Organisationstheorie, Stuttgart: Kohlhammer.

Waldkirch, R. W. (2008): The Shareholder vs Stakeholder Debate Reconsidered. Wittenberg Centre for Global Ethics, Diskussionspapier Nr. 2/2008, Wittenberg: Zentrum für globale Ethik.

Wegner, G. (2012): Ökonomischer Liberalismus als politische Theorie, Tübingen: Mohr Siebeck. Weise, P./Brandes, W./Eger, T./ Kraft, M. (2005): Neue Mikroökonomie, 5. Aufl., Heidelberg: Physica.

Wieland, J. (2001): Eine Theorie der Governanceethik, in: Zeitschrift für Wirtschafts- und Unternehmensethik, Jg. 2/Heft 1, 8-36.

Wieland, J. (2007): Die Ethik der Governance, 5. Aufl., Marburg: Metropolis-Verlag.

Wijnberg, N. M. (2000): Normative Stakeholder Theory and Aristotle: The Link Between Ethics and Politics, in: Journal of Business Ethics, Vol 25/No 4, 329-342.

Will, M. G./Hielscher, S. (2014): How Do Companies Invest in Corporate Social Responsibility? An Ordonomic Contribution for Empirical CSR Research, in: Administrative Sciences, Vol. 4/ No. 3, 219-241.

Witt, U. (1987): Individualistische Grundlagen der evolutorischen Ökonomik, Tübingen: Mohr Siebeck.

Zinkin, J. (2004): Maximising the Licence to Operate, in: Journal of Corporate Citizenship, No. 14, 67-80. 